\title{
Features of Control Processes in ORganizational-Technical (TECHNOLOGICAL) SYSTEMS OF CONTINUOUS TYPE
}

\author{
Submitted: $28^{\text {th }}$ February 2020; accepted: 17 ${ }^{\text {th }}$ August 2020
}

\author{
Igor Korobiichuk, Anatoliy Ladanyuk, Regina Boiko, Serhii Hrybkov
}

DOI: 10.14313/JAMRIS/4-2020/39

\begin{abstract}
Technological complexes of various industries are characterized by certain modes of operation (technological regulations), which correspond to the set of variables of different nature, which have a high-dynamics of change and determine the main technical and economic performance of the object. The aim of the research is to identify information software approaches to support decision-making in organizational-technical (technological) systems. Research results are obtained through grouping, generalization and comparison methods. The scientific significance of the results are to determine the objective need to use intelligent decision support subsystems to quickly manage complex organizational-technical systems based on both: clear and formalized data and knowledge and high-quality fuzzy estimates.
\end{abstract}

Keywords: sugar production, control object, technological monitoring, automated complexes, uncertainty of information, neuro-fuzzy networks

\section{Introduction}

For today information technologies have radically Organizational-technical (technological) systems of continuous type are distinguished by the fact that for the implementation of technological processes they support significant flows of raw materials, energy and energy resources, which in turn generates significant amounts of information that must be processed promptly to obtain effective management actions during preset operating periods, which may be weeks, months, seasons, etc [1].

Most food, processing, chemical and other industries operate in this mode. At the same time an improvement of information support significantly changes the modes of operation, increasing the resource- and energy efficiency of the enterprise [2-4].

One of the trends in the development of modern theory and practice of managing complex objects is the allocation in a separate class of the organizational-technical (technological) systems (OTS), which include both technological complexes, and the enterprise as a whole [5-8]. In fact, the most effective approach to ensuring the operational management of OTS is the development and use of intelligent systems of various levels and purposes. In the structure of intelligent control systems (ICS) one can use the most advanced methods and methods of forming control actions at different levels of the hierarchy: on the lower (executive) the standard automatic regulators function, the most used is the PID-regulator, which in recent years is supplemented by fuzzy (logic) regulators, which use, for example, production rules such as "IF ... THEN". In the general structure of the ICS, there are necessarily databases and knowledge bases, for which the technological monitoring subsystems are used and facilities for the operative assessment of the object's state [9], and for the identification of the interconnections between the process variables - neural network.

Technological complexes of various industries are characterized by certain modes of operation (technological regulations), which correspond to the set of variables of different nature, which have a highdynamics of change and determine the main technical and economic performance of the object.

The aim of the research is to identify information software approaches to support decision-making in organizational-technical (technological) systems based on clear and formalized data and knowledge, as well as high-quality fuzzy estimates.

\section{Materials and Methods of Research}

The authors investigated the works of [5-9] devoted to information software approaches to support decision-making in organizational-technical (technological) systems.

Tool environment Matlab and the Fuzzy Logic Toolbox extension (fuzzy logic package) were used, which includes the subsystem for the development of neuro-fuzzy structures ANFIS (Adaptive-NetworkBased Fuzzy Inference System).

Neuro-fuzzy networks use differentiated realizations of triangular norms (multiplication and probabilistic OR), as well as smooth membership functions. This allows to apply fast neural network learning algorithms to configure neural-fuzzy networks based on reverse error propagation method.

The architecture and rules for each layer of the ANFIS network are described below.

ANFIS implements Sugeno's fuzzy inference system as a five-layer direct propagation neural network. The purpose of the layers is as follows: the first layer - is the terms of the input variables; the second layer - antecedents (parcels) of fuzzy rules; the third layer 
is the normalization of the degrees of implementation of the rules; the fourth layer - the conclusion of the rules; fifth layer - aggregation of the result obtained by different rules.

Network inputs to a separate layer are not highlighted. For the linguistic evaluation of the input variable $x_{1}-3$ terms are used, for the variable $x_{2}-3$ terms, for the variable $x_{3}-3$ terms.

The following notations are required for further presentation: $x_{1}, x_{2}, \ldots, x_{n}$ - network inputs; $y$ - network output; $R 1$ : IF $x_{1}=a_{1, r}$ AND...AND $x n=a_{n, r}$ THEN $y=b_{0, r}+b_{1, r} x_{1}+\ldots+b_{n, r} x n-$ fuzzy rule with ordinal number $\mathrm{r}$; $\mathrm{m}$ - number of rules, $r=\overline{1, m} ; a_{n, r}$ - fuzzy term with membership function $\mu_{r}\left(x_{i}\right)$, used for linguistic evaluation of a variable $x_{i}$ in r-thrule $(r=\overline{1, m}, i=1, n) ; b_{q r}$ - real numbers in the output of r-thrule $(r=\overline{1, m}), q=\overline{0, n)}$.

The ANFIS network works like this.

Layer 1. Each node of the first layer represents one term with a bell-shaped membership function. The network inputs $x_{1}, x_{2}, \ldots, x_{n}$ are only connected to their thermals. The number of nodes in the first layer is equal to the sum of the power of the term-sets of the input variables. The output of the node is the degree of belonging to the value of the input variable corresponding to the fuzzy term (1).

$$
\mu_{r}\left(x_{i}\right)=\frac{1}{1+\left|\frac{x_{i}-c}{a}\right|^{2 b}}
$$

where: $a, b$ and c - options for configuring membership functions.

Layer 2. The number of nodes in the second layer is equal to $\mathrm{m}$. Each node in this layer corresponds to one fuzzy rule. The node of the second layer is connected to those nodes of the first layer that form the antecedents of the corresponding rule. Therefore, each node of the second layer can receive from 1 to $n$ input signals. The output of the node is the degree of execution of the rule, which is calculated as the product of the input signals. Denote the outputs of the nodes of this layer by $\tau_{r}, r=\overline{1, m}$.

Layer 3. The number of nodes in the third layer is also equal to $\mathrm{m}$. Each node in this layer calculates the relative degree of implementation of the fuzzy rule (2).

$$
\tau_{r}^{*}=\frac{\tau_{r}}{\sum_{j=\overline{1, m}} \tau_{j}}
$$

Layer 4. The number of nodes of the fourth layer is also equal to $\mathrm{m}$. Each node is connected to one node of the third layer. The fourth layer node calculates the contribution of one fuzzy rule to the network output:

$$
y_{r}=\tau_{r}^{*} \times\left(b_{0, r}+b_{1, r} x_{1}+\ldots+b_{n, r} x_{n}\right)
$$

Layer 5. A single node in this layer summarizes the contributions of all the rules:

$$
y=y_{1}+\ldots y_{\mathrm{r}} \ldots+y_{\mathrm{m}}
$$

Typical neural network training procedures can be used to set up an ANFIS network because it uses only differentiated functions. Typically, a combination of gradient descent is used in the form of algorithm of reverse error propagation and method of least squares. The algorithm of reverse error propagation adjusts the antecedents of the rules, that is, the membership functions.

The method of least squares estimates the coefficients of the rules because they are linearly related to the output of the network.

Each iteration of the tuning procedure is performed in two steps. In the first stage, a training sample is submitted to the inputs, and the optimum parameters of the nodes of the fourth layer are determined by the deviation between the desired and actual network behavior by the iterative method of least squares.In the second step, the residual deviation is transmitted from the output of the network to the inputs, and the parameters of the first layer nodes are modified by the method of reverse error propagation.At the same time, the coefficients of conclusions of the rules found in the first stage do not change.The iterative tuning procedure continues until the residual exceeds the preset value.

\section{The Results of Research}

Complex technological objects, which are part of the OTS, always function in the conditions of intense perturbations and changing characteristics of the external environment, which leads to the need to take into account significant uncertainties and their inadequate decisions and the formation of management actions that do not correspond to the production situation and lead to significant risks.

Considering the information provision of management processes it is advisable to take as a basis subsystems of decision support, in particular, in intelligent control systems where management actions are taken or controlled by the person who makes the decision (PMD). In this regard, the uncertainty is interpreted as incomplete, vague, obscure or ambiguous information about the object and its state in different operating modes. At the same time, the preparation of management actions is clearly related to risk as a certain possibility of obtaining a planned result, while the risk and uncertainty have the same essence and are measured in some units, for example, in percent, that is, uncertainty can become a risk in the implementation of management actions, and the implementation of a risky decision can lead to uncertainty. Thus, uncertainty as a phenomenon - fuzzy and blurry, contradictory descriptions of the object and production situations, insufficient and mutually exclusive information. When managing complex objects, it is necessary to take into account force majeure events that arise independently of the will and human consciousness and change the course of the control processes. It should also be taken into account that the actions of PMD, in it turn, can lead to uncertainty 
- incompetence or unjustified decision without consideration of the problem situation and lack of time.

In the management process, uncertainty at any level leads to risk, and the greater the uncertainty, the higher the risks.

The technical literature gives enough places to classify variants and types of uncertainty of information, although in many ways it depends on the object of management and the peculiarities of its operation [10-16]. First of all, it is advisable to distinguish between two main classes: structural and parametric uncertainty and each of them, for the tasks of controlling and controlling the state of the object is complemented by many indicators, for example:

- low accuracy of operational information about the state of the object (low accuracy of technical means for receiving and processing of data, failure of communication channels, transmission latency between levels of management, etc.);

- inaccuracy of object models and description of information arrays, in particular systemic errors: incorrectly carried out decomposition (allocation of subsystems), relating both to object and task of management, linearization of models and their sampling;

- obscurity of information due to the difficulties of formalizing a number of indicators, the use of linguistic variables and "soft" calculations;

- the presence of a large number of management criteria (optimization) and the need to take into account a variety of constraints of different nature;

- decision-making in multilevel hierarchical systems of an iterative nature with the need to solve numerous auxiliary tasks of coordinating decisions between levels.

Finally, with the current development of information technologies separately identified uncertainty as information resource (knowledge) that in developed countries in the gross domestic product (GDP) is up to $30 \%$ due to the creation of value added. To characterize information resources, the term "content" is used as the content of a particular resource, which is considered outside the forms of its material representation - audio and video texts, individual images, verbal texts, symbols, etc.In a modern approach, the content may not be structured (Webcontent) or structured, for example, at the enterprise - tables, databases and knowledge bases, data warehouses, content displayed in a certain form - informative resource as a collection of data, information and knowledge that accumulate in the process of human activity and the operation of a particular object. In the information idea (K. Shennon, 1948), the amount of information is considered as a measure of certainty or uncertainty of the state of the system, which is used without mediation in the management process. Determining this uncertainty as entropy, it is assumed that obtaining information (its increase) reduces entropy. The measured uncertainty, in fact, the risk, is so different from the uncertain that it is not generally uncertain [13-16].

When managing technological complexes in the OTS class, the presentation of data and knowledge in the form of fuzzy sets with different membership functions becomes of particular importance. At the same time, the level of uncertainty is unambiguously connected with the value of the membership function, the decrease of which characterizes the deterioration of the quality of management decisions based on the fuzzy conclusion with the operations of merging and crossing fuzzy sets. The most important are the fuzzy rules "IF...THEN..." which form control poor (vague) defined objects. The comparison of the fuzzy set A with some base $A^{*}$ whose level of fuzziness is zero are used. To bring the comparison result in a range from 0 to 1 , the formula [14-18] is used (5):

$$
\rho\left(A, A^{*}\right)=\frac{2}{n} d\left(A, A^{*}\right)
$$

where:

$\rho\left(A, A^{*}\right)$ - the uncertainty level of the fuzzy set $\mathrm{A}$ in comparison with the fuzzy set $\mathrm{A}^{*}$;

$d\left(A, A^{*}\right)$ - Hamming's line distance between sets $\mathrm{A}$ and $\mathrm{A}^{*}(6)$.

$$
d\left(A, A^{*}\right)=\sum_{i=1}^{n}\left|\mu_{A}\left(x_{i}\right)-\mu_{A^{\sharp}}\left(x_{i}\right)\right|
$$

where:

$$
\begin{aligned}
& \mu_{A}\left(x_{i}\right), \mu_{A^{\sharp}}\left(x_{i}\right) \text { - the functions of the membership of the } \\
& \text { set A and } A^{*} \text {; } \\
& n \text { - number of items compared in sets. }
\end{aligned}
$$

As can be seen from Fig. 1 - result of unions of fuzzy sets, the uncertainty of the output (result) of the set increases [15-16].

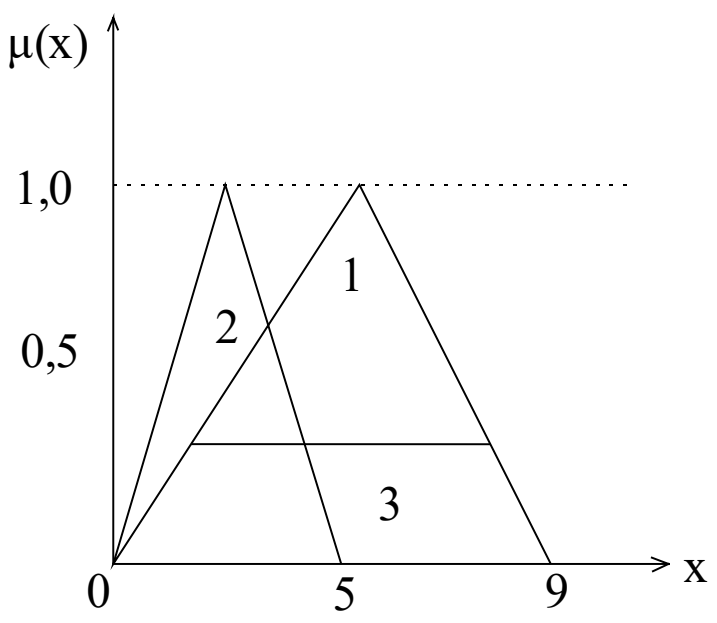

a) 


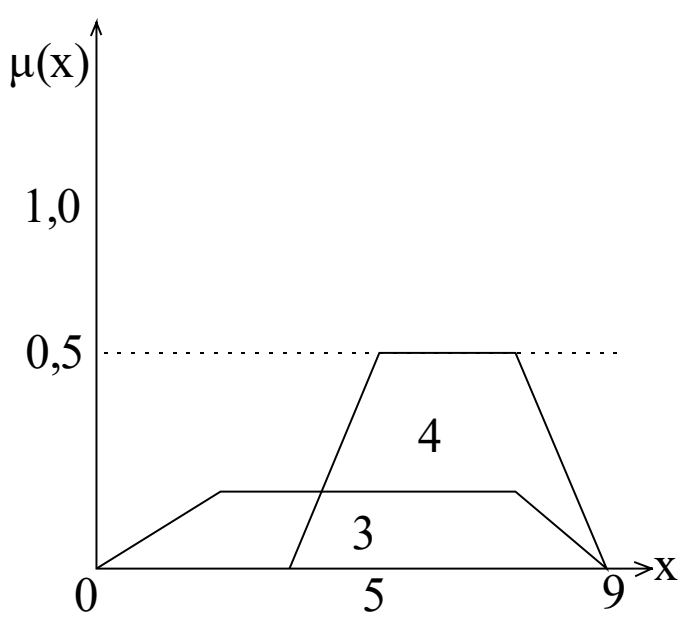

b)

Fig. 1. Functions of membership: $a-$ initial, $b$ - result of association

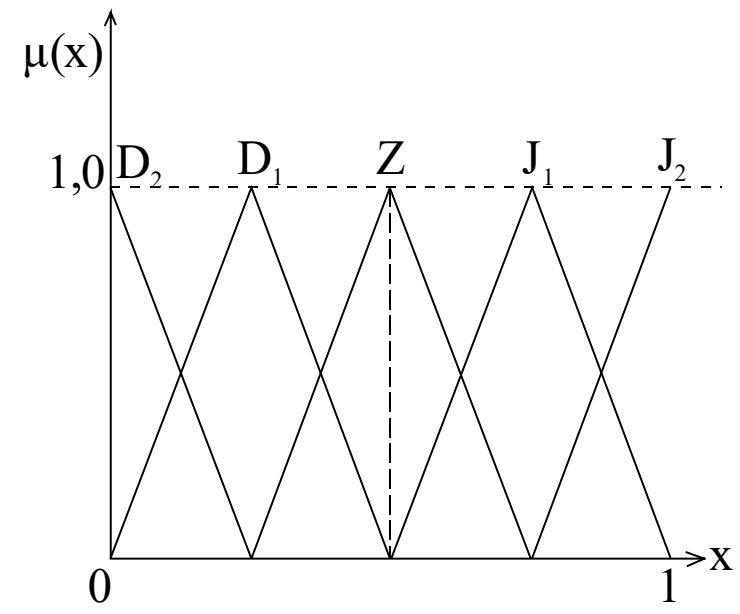

Fig. 2. Term of the set of linguistic variable "cost change"

Technological complexes of various industries are characterized by certain operating regimes (technological regulations), which correspond to the set of variables of different nature - temperature, level, pressure, etc., and in many cases material flows are acquired (raw materials, intermediate liquid flows, pairs, etc.) which have a high dynamic change and determine the main technical and economic performance of the object. An approach based on fuzzy logic is increasingly used to evaluate and manage material flows. For example, Fig. 2 and Fig. 3 show the functions of the inclusion of linguistic variables "cost change" and "cost".

On Fig. 2 marked: $D_{2}$ - "significantly reduce", $D_{1}-$ "reduce somewhat", $Z$ - "do not change", $J_{1}$ - "increase somewhat", $J_{2}$ - "significantly increase". On Fig. 3 marked: $T_{M}$ - "small", $T_{C}$ - "medium", $T_{B}$ - "big". On Fig. 2 and Fig. 3 the membership functions have a triangular shape, and in specific cases there could be another form, for example, exponential.

One of the most powerful sources of information for decision-making tasks is time rows [19-20], as a set of consistent observational results. Time rows, as a rule, arise as a result of measuring a certain

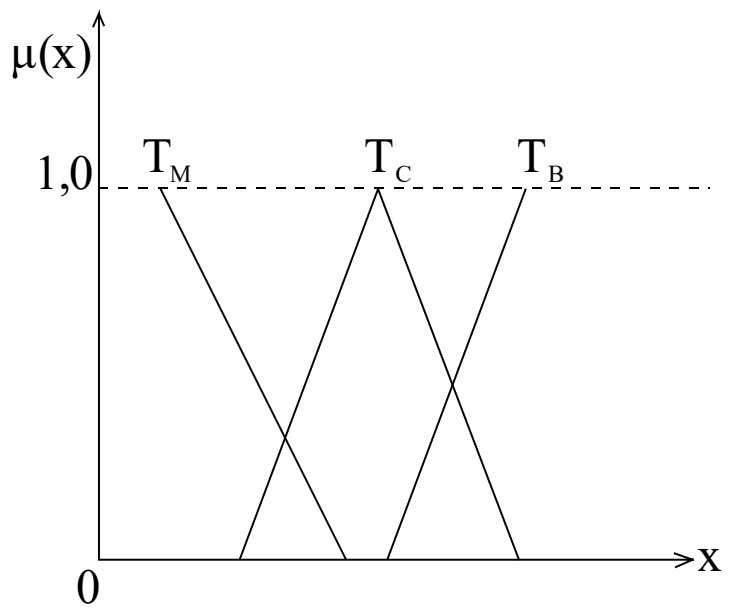

Fig. 3. Term of the set of linguistic variable "cost change"

indicator, for example, technological variables. In the time rows, for each reference, the measurement time or measurement number must be specified in order. The time rows significantly differ from the sample by the fact that it is necessary to take into account the connection of measurements with time. Time rows consist of two elements: the time for which the time values are given, and the actual numerical values of the indicator being analyzed (row level). Time rows allow objectively evaluate the state of an object, analyze its behavior, perform forecasting (extrapolation), determine the prospects for its development, identify characteristic manifestations of the behavior of the object (patterns), to form a base of precedents. The solution of the above tasks will, of course, sharply increase the efficiency of decision-making [21]. Time rows filtering have two components in its composition: a useful signal and noise, whose parameters are unknown and whose intensity is situational depending on various factors [22]. An effective approach for filtering time rows in which unstable noise characteristics are is the use of wavelet analysis [19-24].

The problem of identifying production processes based on a neuro-fuzzy network (NFN) is relevant, based on the conceptual foundations of the complexity of interconnections between input and output variables.

In this case, we consider the technological complex of sugar production, the parametric scheme of which is shown in Fig. 4.

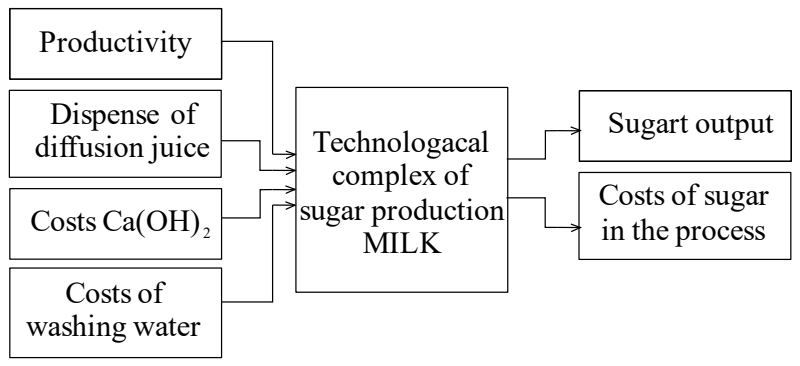

Fig. 4. Parametric scheme of the main flows 
We propose a solution for this problem by solving two problems - two steps respectively. For correct representation of data and based on the peculiarities of the implementation of the algorithm of the logical Sugeno's conclusion will be constructed two neuro-fuzzy networks. Solving this problem is done using the MatLab toolkit. In order to realize the solution of this problem, it is proposed to use the internal subsystem of Matlab environment - the subsystem of the development of neuro-fuzzy structures ANFIS. ANFIS is the Adaptive-Network-Based Fuzzy Inference System abbreviation - an adaptive network of fuzzy output. ANFIS is one of the first variants of hybrid neuro-fuzzy networks - a special type of direct propagation of the neural network. The architecture of a neuro-fuzzy network is isomorphic to a fuzzy knowledge base. In neuro-fuzzy networks, differentiated realizations of triangular norms are used (multiplication and probabilistic OR), as well as smooth membership functions. This allows the use of fast neural network training algorithms based on the method of reverse error propagation to set up neuro-fuzzy networks. The following describes the architecture and rules for the operation of each layer of the ANFIS network.

ANFIS implements Sugeno's fuzzy detection system in the form of a five-layer neural network of direct signal propagation. Assign layers as follows:

- the first layer -the terms of the input variables;

- the second layer - antecedents (parcels) of fuzzy rules;

- the third layer - normalization of the degree of execution of the rules;

- the fourth layer - the conclusion of the rules;

- the fifth layer - the aggregation of the result obtained by different rules.

To explain the content of the feedback surface of the knowledge base, we introduce the following designations of the graphs: Input 1 - the productivity of the plant; Input 2 - pump diffusion juice; Input 3 consumption of lime milk; Input 4 - flow of flushing water; Output - sugar output.

At the second stage of the problem's solution, namely, to identify the main cause-effect relationships that allow to analyze the impact of such factors as productivity, discharge of diffusion juice, the cost of lime milk, the cost of washing water on sugar loss in the production process (Fig. 5).

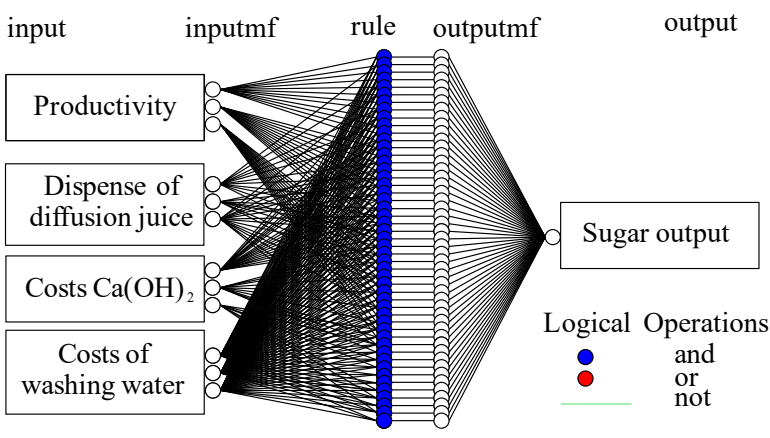

Fig. 5. The structure of the neuro-fuzzy network to identify the causal relationships of assessing the loss of sugar in the production process
Information for time series is obtained from real enterprises for the same time intervals, namely for the last 2 years. Time series from six months to two years were used in the construction of neural networks and their testing.

The above approach allows to simplify the work of expert people to identify the main relationships between the input and output variables of any technological complex, to generate information material for person who makes decisions (PMD). Solving the problem of identification on the basis of neuron-fuzzy approach has shown the possibility of establishing causal relationships between input and output variables processes sugar production in the form of fuzzy rules [25].

An effective modern approach to the creation of information management systems is a combination of methods for accounting both uncertainties and risks [22]. A particular importance is the formation of management (managerial) solutions in large systems, where the choice of options for their implementation is calculated by economic efficiency within the financial capabilities of the enterprise. The formalization of the choice of decision options is based on the introduction of an evaluation target function, when each alternative $x_{i}$ PMD attributes a probabilistic result $P\left(x_{i}\right)$ that characterizes all the consequences of this solution. There are a number of approaches in the technical literature, for the implementation of which various criteria are used, for example:

- maximum mathematical expectation (hope) of gain, when the ODA can actually be estimated on the basis of the probability distribution of the state of the object and the environment;

- minimal dispersion, which reduces the risk of obtaining a slight gain with a significant mathematical expectation of the range of its change;

- the marginal level of gain at the existing interval;

- the most probable result when it is possible to obtain all the necessary information for decision-making;

- minimum average risk, when each of its values corresponds to the state of the environment.

In order to use the statistical approach, a significant representative sample of observations or expert knowledge must be available for the formation of control actions with changing characteristics of the object and the environment.

Modeling and testing were performed using Matlab and Fuzzy Logic Toolbox extension (fuzzy logic package), but it is not possible to include it in real OTS control systems. The authors created software modules on C\# language in which the proposed methods and neuro-fuzzy structures ANFIS are fully implemented. Created software modules can be included in existing information systems and those that are being created. Due to the fact that the created software modules are created on C\#, integration goes smoothly. 


\section{Conclusion}

The scientific significance of the results are to determine the objective need to use intelligent decision support subsystems to quickly manage complex OTS based on both: clear and formalized data and knowledge and high-quality fuzzy estimates. Uncertainty of information is highlighted, technological monitoring values and the possibility of using neural networks to assess the status of control objects are shown.

For efficient management of the OTS of different nature and purpose, the priority task is to create the necessary information support for the assessment of which the information resource (content) is used as its content beyond the forms of its material representation.

It is shown that the formation of management actions in the OTS is unambiguously connected with the uncertainty of the information and the caused risks, the types of uncertainty of information are given.

For the operational management of complex OTS the objective necessity of using intelligent subsystems of decision support based on both clear formalized data and knowledge and qualitative fuzzy evaluations is determined.

Are given examples of use for the estimation of fuzzy knowledge of membership functions, and for the identification of the state of an object is an adaptive neuro-fuzzy network.

In the conditions of long functioning of technological complexes in the OTS, a number of criteria for statistical analysis have been selected for the assessment of technical and economic indicators.

\section{AUTHORS}

Igor Korobiichuk* - ŁUKASIEWICZ Research Network - Industrial Research Institute for Automation and Measurements PIAP, Jerozolimskie 202, 02-486 Warsaw, Poland, e-mail: igor.korobiichuk@piap.lukasiewicz.gov.pl

Anatoliy Ladanyuk - National University of Food Technologies, 68 Volodymyrska Street, 01033, Kyiv, Ukraine, e-mail: ladanyuk@ukr.net.

Regina Boiko - National University of Food Technologies, 68 Volodymyrska Street, 01033, Kyiv, Ukraine, e-mail: rela@ukr.net.

Serhii Hrybkov - National University of Food Technologies, 68 Volodymyrska Street, 01033, Kyiv, Ukraine, e-mail: sergio_nuft@nuft.edu.ua.

${ }^{*}$ Corresponding author

\section{REFERENCES}

[1] I. Korobiichuk, A. Ladanyuk, L. Vlasenko and N. Zaiets, "Modern Development Technologies and Investigation of Food Production Technological Complex Automated Systems". In: Pro- ceedings of the 2nd International Conference on Mechatronics Systems and Control Engineering - ICMSCE 2018, 2018, 52-56, DOI: $10.1145 / 3185066.3185075$.

[2] I. Korobiichuk, N. Lutskaya, A. Ladanyuk, S. Naku, M. Kachniarz, M. Nowicki and R. Szewczyk, "Synthesis of Optimal Robust Regulator for Food Processing Facilities". In: R. Szewczyk, C. Zieliński and M. Kaliczyńska (eds.), Automation 2017, vol. 550, 2017, 58-66, DOI: 10.1007/978-3-319-54042-9_5.

[3] V. Tregub, I. Korobiichuk, O. Klymenko, A. Byrchenko and K. Rzeplińska-Rykała, "Neural Network Control Systems for Objects of Periodic Action with Non-linear Time Programs". In: R. Szewczyk, C. Zieliński and M. Kaliczyńska (eds.), Automation 2019, vol. 920, 2020, 155-164, DOI: 10.1007/978-3-030-13273-6_16.

[4] V. Sidletskyi, I. Korobiichuk, A. Ladaniuk, I. Elperin and K. Rzeplińska-Rykała, "Development of the Structure of an Automated Control System Using Tensor Techniques for a Diffusion Station". In: R. Szewczyk, C. Zieliński and M. Kaliczyńska (eds.), Automation 2019, vol. 920, 2020, 175-185, DOI: 10.1007/978-3-030-13273-6_18.

[5] A. P. Ladanyuk, N. M. Lutska, V. D. Kishenko, L. O. Vlasenko and V. V. Ivaschuk, Methods of conventional theory of management, Lira: Kyiv, 2018 (in Ukrainian).

[6] J. Stahre, L. Mårtensson (eds.), Proceedings of 8th IFAC Symposium on Automated Systems Based on Human Skill and Knowledge, Elsevier, 2003.

[7] M. P. Kazmierkowski, "Integration Technologies for Industrial Automated Systems", IEEE Industrial Electronics Magazine, vol. 1, no. 1, 2007, 51-52, DOI: $10.1109 /$ MIE.2007.357179.

[8] A. Colombo, T. Bangemann, S. Karnouskos, J. Delsing, P. Stluka, R. Harrison, F. Jammes, and J. L. Lastra (eds.), Industrial Cloud-Based CyberPhysical Systems: The IMC-AESOP Approach, Springer International Publishing, 2014, DOI: 10.1007/978-3-319-05624-1.

[9] W. Q. Yan, Introduction to Intelligent Surveillance: Surveillance Data Capture, Transmission, and Analytics, Springer International Publishing, 2019, DOI: $10.1007 / 978-3-030-10713-0$.

[10] T. O. Prokopenko and A. P. Ladanyuk, Information technology management organizational and technological systems, Vertikal: Cherkasi, 2015 (in Ukrainian).

[11] J. Yu, Y. Li, M. Chen, B. Zhang and W. Xu, "Decision-theoretic rough set in lattice-valued decision information system", Journal of Intelligent \& Fuzzy Systems, vol. 36, no. 4, 2019, 32893301, DOI: $10.3233 / J I F S-172111$. 
[12] S. Hrybkov and H. Oliinyk, "Modeling of the decision support system structure in the planning and controlling of contracts implementation", Ukrainian Journal of Food Science, vol. 3, no. $1,2015,123-130$.

[13] T. Bakshi, B. Sarkar and S. K. Sanyal, "An Evolutionary Algorithm for Multi-criteria Resource Constrained Project Scheduling Problem based On PSO", Procedia Technology, vol. 6, 2012, 231-238,

DOI: 10.1016/j.protcy.2012.10.028.

[14] S. Hrybkov, H. Oliinyk and V. Litvinov, "Weboriented decision support system for planning agreements execution", Eastern-European Journal of Enterprise Technologies, vol. 3, no. 2 (93), 2018, 13-24,

DOI: $10.15587 / 1729-4061.2018 .132604$.

[15] N. M. Lutska and A. P. Ladanyuk, Optimal and robust control systems for technological objects, Lira: Kyiv, 2015 (in Ukrainian).

[16] F. Jabari, S. Nojavan, B. Mohammadi-Ivatloo, H. Ghaebi and M.-B. Bannae-Sharifian, "Robust Unit Commitment Using Information Gap Decision Theory". In: B. Mohammadi-ivatloo and M. Nazari-Heris (eds.), Robust Optimal Planning and Operation of Electrical Energy Systems, 2019, 79-93,

DOI: 10.1007/978-3-030-04296-7_5.

[17] H.-J. Zimmermann, "Fuzzy set theory", WIREs Computational Statistics, vol. 2, no. 3, 2010, 317-332, DOI: $10.1002 /$ wics.82.

[18] W. Pedrycz and P. Rai, "Collaborative clustering with the use of Fuzzy C-Means and its quantification", Fuzzy Sets and Systems, vol. 159, no. 18, 2008, 2399-2427,

DOI: $10.1016 /$ j.fss.2007.12.030.
[19] E. Lughofer, A.-C. Zavoianu, M. Pratama and T. Radauer, "Automated Process Optimization in Manufacturing Systems Based on Static and Dynamic Prediction Models". In: E. Lughofer and M. Sayed-Mouchaweh (eds.), Predictive Maintenance in Dynamic Systems, 2019, 485-531, DOI: 10.1007/978-3-030-05645-2_17.

[20] J. Beran, Mathematical Foundations of Time Series Analysis: A Concise Introduction, Springer International Publishing, 2017, DOI: 10.1007/978-3-319-74380-6.

[21] N. T. Son and D. T. Anh, "Discovering Time Series Motifs Based on Multidimensional Index and Early Abandoning". In: N.-T. Nguyen, K. Hoang and P. Jędrzejowicz (eds.), Computational Collective Intelligence. Technologies and Applications, 2012, 72-82, DOI: $10.1007 / 978-3-642-34630-9 \_8$.

[22] A. Tapinos and P. Mendes, "A Method for Comparing Multivariate Time Series with Different Dimensions", PLOS ONE, vol. 8, no. 2, 2013, DOI: $10.1371 /$ journal.pone.0054201.

[23] L. Wei and E. Keogh, "Semi-Supervised Time Series Classification". In: Proceedings of the 12th ACM SIGKDD International Conference on Knowledge Discovery and Data Mining, 2006, 748-753, DOI: $10.1145 / 1150402.1150498$.

[24] S. Hira and P. S. Deshpande, "Data Analysis using Multidimensional Modeling, Statistical Analysis and Data Mining on Agriculture Parameters", Procedia Computer Science, vol. 54, 2015, 431-439, DOI: 10.1016/j.procs.2015.06.050.

[25] J. Burgess, Wavelets: Principles, Analysis and Applications, Nova Science Publishers, Incorporated, 2018. 